\title{
Model Reduction of Power Systems with Preservation of Slow and Poorly Damped Modes
}

\author{
Giordano Scarciotti \\ Department of Electrical and Electronic Engineering \\ Imperial College London \\ London, United Kingdom \\ Email: gs3610@imperial.ac.uk
}

\begin{abstract}
In this paper a recently proposed variation of the Krylov subspace method for model reduction is applied to power systems. The technique allows to easily enforce constraints on the reduced order model. Herein this is used to preserve the slow and poorly damped modes of the systems in the reduced order model. We analyze the role that these modes have in obtaining a good approximation and we show that the order of the reduced model can be decreased if the "right" modes are preserved. We validate the theory on the 68-Bus, 16-Machine, 5-Area benchmark system (NETS-NYPS).
\end{abstract}

Index Terms-Dynamic equivalents, model reduction of power systems, power system simulation, coherency.

\section{INTRODUCTION}

Simulation of power systems for dynamic analysis, trajectory sensitivity analysis and control design is a computationally intensive task. The mathematical models used to describe power systems can easily reach hundreds of differential equations. Thus, the need to determine less complex yet meaningful descriptions (in a sense to be specified) is a problem which is central to modern research. This problem, called model reduction in the control community [1] and, some times, dynamic equivalencing in the power system community [2], consists in finding a simplified mathematical model which maintains some key properties of the original model. In this equivalent model we distinguish between a study area, the description of which is maintained in full detail, and an external area, consisting of the remaining part of the network, which is reduced. Historically, coherency-based methods have been used in model reduction of power systems, see e.g. [3], [4], [5], [6]. These are based on the physical properties of the electrical machines connected to the network. The idea is to find coherent generators, i.e. machines which behave similarly when the same input is applied. Once coherent generators are identified a dynamic equivalent generator is used to replace them. In recent years, the power system community has started to be interested in reduction techniques based on mathematical properties instead of physical ones. One of the reasons of this interest is the flexibility of having a reduction technique that is not based on the physics of the generators and, as a consequence, the possibility of reducing networks with renewable energy sources. Among these methods, balanced truncation and Krylov projectors have been successfully used in power systems reduction, see e.g. [7], [2], [8]. One of the drawbacks of the techniques based on Krylov projections, also called moment matching methods, is the difficulty in enforcing or preserving important properties of the system to be reduced. To be of any use, it is of paramount importance that the reduced order model of a power system preserves slow modes and poorly damped modes. Poorly damped modes, also called electromechanical modes [9], are important in the small-signal stability analysis of a power system since they are responsible for most of the oscillating behavior. Previous attempts to maintain these modes are essentially ad hoc since with the classical Krylov methods it is very hard to assign a certain set of eigenvalues. However, following the ideas in [10], this problem has been alleviated. Moreover, the new interpretation given in [10] led to further developments in the model reduction field, see e.g. [11], [12], [13] and [14], which may be of interest for the model reduction of power systems. The contribution of this paper is twofold. The first one is showing that it is possible with the moment matching technique presented here to assign arbitrary eigenvalues to the reduced order model and this is exploited to maintain slow and poorly damped modes. The second one is to disprove the belief that it is necessary to increase the order of the reduced order model to improve the quality of the approximation of the system. This is wrong in two ways: firstly, to improve the approximation it may be sufficient to select a different set of eigenvalues to be preserved in the reduced order model; secondly, increasing the order of the reduced model gives no guarantee of improving significantly the quality of the approximation if the wrong set of eigenvalues are preserved.

The rest of the paper is organized as follows. In Sections II-A and II-B a brief presentation of the moment matching method is given for single-input, single-output (SISO) systems and multi-input, multi-output (MIMO) systems, respectively. In Section III-A we describe the model used in our analysis. In Section III-B a 68-Bus, 16-Machine, 5-Area system is used to illustrate the results of the paper.

\section{Model Reduction By Moment Matching}

\section{A. SISO Systems}

Consider a linear, single-input, single-output, continuoustime, system described by the equations

$$
\dot{x}=A x+B u, \quad y=C x,
$$

with $x(t) \in \mathbb{R}^{n}, u(t) \in \mathbb{R}, y(t) \in \mathbb{R}, A \in \mathbb{R}^{n \times n}, B \in \mathbb{R}^{n \times 1}$ and $C \in \mathbb{R}^{1 \times n}$. Let $W(s)=C(s I-A)^{-1} B \in \mathbb{R}$ be the associated transfer function and assume that (1) is minimal, i.e. controllable and observable.

Definition 1: Let $s_{i} \in \mathbb{C}$, with $s_{i} \notin \sigma(A)$. The 0-moment of system (1) at $s_{i}$ is the complex number $\eta_{0}\left(s_{i}\right)=C\left(s_{i} I-\right.$ 
$A)^{-1} B$. The $k$-moment of system (1) at $s_{i}$ is the complex number $\eta_{k}\left(s_{i}\right)=\frac{(-1)^{k}}{k !}\left[\frac{d^{k}}{d s^{k}} W(s)\right]_{s=s_{i}}$, with $k \geq 1$ integer.

In [10] (see also [15] and [16]), a characterization of the moments of system (1) has been given in terms of the solution of a Sylvester equation as follows.

Lemma 1: [10] Consider system (1), $s_{i} \in \mathbb{C}$ and suppose $s_{i} \notin \sigma(A)$, for all $i=1, \ldots, \eta$. There exists a one-to-one relation between the moments $\eta_{0}\left(s_{1}\right), \ldots, \eta_{k_{1}-1}\left(s_{1}\right), \ldots$, $\eta_{0}\left(s_{\eta}\right), \ldots, \eta_{k_{\eta}-1}\left(s_{\eta}\right)$ and the matrix $C \Pi$, where $\Pi \in \mathbb{R}^{n \times \nu}$ is the unique solution of the Sylvester equation

$$
A \Pi+B L=\Pi S,
$$

with $S \in \mathbb{R}^{\nu \times \nu}$ any non-derogatory matrix with characteristic polynomial

$$
p(s)=\prod_{i=1}^{\eta}\left(s-s_{i}\right)^{k_{i}}
$$

where $\nu=\sum_{i=1}^{\eta} k_{i}$, and $L \in \mathbb{R}^{1 \times \nu}$ is such that the pair $(L, S)$ is observable.

Finally, as shown in [10], the family of systems

$$
\dot{\xi}=(S-G L) \xi+G u, \quad \psi=C \Pi \xi,
$$

with $G \in \mathbb{R}^{\nu \times 1}$ any matrix such that $\sigma(S) \cap \sigma(S-G L)=$ $\emptyset$, contains all the models of dimension $\nu$ interpolating the moments of system (1) at the eigenvalues of the matrix $S$. Hence, we say that system (4) is a model of (1) at $S$. System (4) is a reduced order model of system (1) at $S$ if $\nu<n$.

Remark 1: The model reduction technique by moment matching is based on the idea of interpolating a certain number of points $s_{i}$ on the complex plane: a reduced order model is such that its transfer function (and derivatives of this) takes the same values of the transfer function (and derivatives of this) of system (1) at $s_{i}$.

Remark 2: All the models that can be obtained using Krylov projectors are encoded in the family of systems (4). Thus the two approaches are equivalent. The advantage of this formulation is that the family of systems (4) is parametrized in $G$, which allows to set with ease several properties of the reduced order model, as shown in [10]. For instance, setting the eigenvalues of the reduced order model is a trivial task, whereas with the classic Krylov method this is hard.

\section{B. MIMO Systems}

Consider a linear, multi-input, multi-output, continuoustime, system described by (1), with $x(t) \in \mathbb{R}^{n}, u(t) \in \mathbb{R}^{m}$, $y(t) \in \mathbb{R}^{p}, A \in \mathbb{R}^{n \times n}, B \in \mathbb{R}^{n \times m}$ and $C \in \mathbb{R}^{p \times n}$. Let $W(s)=C(s I-A)^{-1} B \in \mathbb{R}^{m \times m}$ be the associated transfer function and assume that (1) is minimal. Let $S \in \mathbb{R}^{\nu \times \nu}$ be any non-derogatory matrix with characteristic polynomial (3) and $L=\left[l_{1} l_{2} \ldots l_{\nu}\right] \in \mathbb{R}^{m \times \nu}, l_{i} \in \mathbb{R}^{m \times 1}, i=1, \ldots, \nu$, be such that the pair $(L, S)$ is observable. Then the moments of the system, namely $\eta_{k}\left(s_{i}\right)=\frac{(-1)^{k}}{k !}\left[\frac{d^{k}}{d s^{k}} W(s)\right]_{s=s_{i}} l_{i}$, are in one-to-one relation with $C \Pi$, with $\Pi \in \mathbb{R}^{n \times \nu}$ unique solution of the Sylvester equation (2).

Let $\hat{W}(s)=H(s I-F)^{-1} G \in \mathbb{R}^{m \times m}$, with $F=S-G L \in$ $\mathbb{R}^{\nu \times \nu}, G \in \mathbb{R}^{\nu \times m}$ and $H=C \Pi \in \mathbb{R}^{p \times \nu}$, be the transfer function of system (4). The family of systems (4) is a model of system (1) at $S$ if $G$ is such that $\sigma(S) \cap \sigma(S-G L)=\emptyset$ and [17], [18]

$$
\frac{(-1)^{k}}{k !}\left[\frac{d^{k}}{d s^{k}} W(s)\right]_{s=s_{i}} l_{i}=\frac{(-1)^{k}}{k !}\left[\frac{d^{k}}{d s^{k}} \hat{W}(s)\right]_{s=s_{i}} l_{i},
$$

for $i=1, \ldots, \nu$. System (4) is a reduced order model of system (1) at $S$ if $\nu<n$.

Remark 3: In the MIMO case, the reduced order model has to satisfy the additional constraints (5). These are called right tangential interpolation conditions [19]. The matrix $G$ can be selected to satisfy these conditions as suggested in [17], however, in this way $G$ cannot be used to enforce additional properties on the reduced order model. In this paper we propose another approach. We use $G$ to set some desired properties, e.g. to assign the eigenvalues, and we use an optimization algorithm to find the corresponding matrix $L$. Due to lack of space we omit this algorithm that will be published in a longer version of this paper.

\section{APPLICATION TO POWER Systems}

\section{A. Power system model}

The classical model, see [20], [21], normally used in the literature of model reduction of power systems, see e.g. [2], [7], [8], is used to describe a power system composed of $n_{m}$-machines and $n_{b}$-bus. The model is described by the differential equations

$$
\begin{aligned}
\dot{\delta}_{i} & =\omega_{i}-\omega_{s}, \\
\frac{2 H_{i}}{\omega_{s}} \dot{\omega}_{i} & =T_{M_{i}}-D_{i}\left(\omega_{i}-\omega_{s}\right)-E_{i}^{2} G_{i i} \\
& -E_{i} \sum_{\substack{j=1 \\
j \neq i}}^{n_{m}}\left(E_{j} G_{i j} \cos \left(\delta_{i}-\delta_{j}\right)+E_{j} B_{i j} \sin \left(\delta_{i}-\delta_{j}\right)\right),
\end{aligned}
$$

with $i=1, \ldots, n_{m}$, where $\delta_{i}$ and $\omega_{i}$ are the rotor angle and angular velocity of the $i$ th machine, respectively, $\omega_{s}$ is the reference angular velocity, $H_{i}$ and $D_{i}$ are the inertia and damping coefficients, respectively, $E_{i}$ is the internal voltage of the machine $i, Y_{i j}=G_{i j}+\iota B_{i j}$ is the admittance between the machines $i$ and $j, G_{i i}$ is the self-conductance of the $i$ th machine and $T_{M_{i}}$ is the mechanical input power.

In the literature on model reduction of power systems, see $e . g$. [2], [7], the study area and the external area are often modeled as two separate entities interconnected each other with $n_{p^{-}}$ tie-lines. However, this is a somewhat strong approximation. In fact, note that if the two power systems, study area and external area, are interconnected then we have a unique large power system and the power flow analysis which defines the parameters of system (6) has to be updated. Instead, in some of the literature the two systems are actually kept separated and the tie-lines are only used to exchange the input and output of the two systems. This gives the considerable simplification that the number of input and output corresponds to the number of tie-lines.

On the contrary, in this paper the division in study area and 


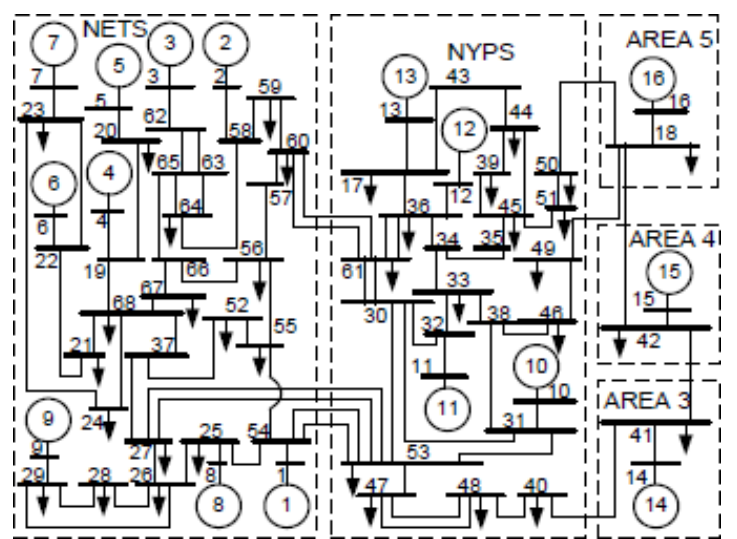

Fig. 1. Line diagram of the 68-bus system.

external area is a pure exercise of labeling. In fact, the whole power system is described by system (6) and since the division in study and external area is arbitrary it can be done over every area of the power systems. This approach has the advantage of improving the fidelity of the simulation of the power system. The drawback is that the number of inputs of the external area is the number of machines of the study area and the number of outputs of the external area is the number of machines of the external area, and vice-versa. However, since with the considered method the dimension of the reduced order model does not depend upon the number of inputs and outputs, a large number of inputs and outputs is not an issue for the technique we are presenting. Thus, if the study area has $s_{m}$-machines and the external area has $e_{m}$-machines, with $s_{m}+e_{m}=n_{m}$, the study area is described by system (6) for $i=1, \ldots, s_{m}$ and $\delta_{j}$, with $j=s_{m}+1, \ldots, s_{m}+e_{m}$, is the input of the study area (output of the external area). The external area is described by the linearization of system (6) around an equilibrium point, namely

$$
\begin{aligned}
{\left[\begin{array}{c}
\Delta \dot{\delta} \\
\Delta \dot{\omega}
\end{array}\right] } & =\left[\begin{array}{cc}
0 & I \\
A_{21} & A_{22}
\end{array}\right]\left[\begin{array}{c}
\Delta \delta \\
\Delta \omega
\end{array}\right]+\left[\begin{array}{c}
0 \\
B_{2}
\end{array}\right] \Delta u, \\
y & =\left[\begin{array}{ll}
C_{1} & 0
\end{array}\right]\left[\begin{array}{c}
\Delta \delta \\
\Delta \omega
\end{array}\right],
\end{aligned}
$$

with some matrices $A_{21}, A_{22}, B_{2}$ and $C_{1}, \Delta \delta_{i}=\delta_{i}-\delta_{i}^{0}$, $\Delta \omega_{i}=\omega_{i}-\omega_{i}^{0}$, with $i=1, \ldots, e_{m}$ and $\Delta u_{j}=u_{j}-u_{j}^{0}=\delta_{j}-$ $\delta_{j}^{0}$, with $j=1, \ldots, s_{m}$, where $\left(\delta^{0}, \omega^{0}, u^{0}\right)$ is an equilibrium point. The remaining quantities are not defined because of lack of space but they can be easily determined applying standard linearization.

\section{B. NETS-NYPS system}

The theory presented in Section II is validated on the interconnected New England test system (NETS) and New York test system (NYPS) 68-bus, 16-machine, 5-area power system shown in Fig. 1, see [9]. The study area, composed of the machines 14,15 and 16 , is interconnected with the bus-lines $18-50,18-49$ and $41-40$ to the external area, composed of the machines from 1 to 13 . The separation in study and external area follows the geographical distribution of the power system and the tie-lines are actual bus-lines of the power system. The system to be reduced has $n=26, m=3, p=13$.

In this section we disprove the common belief that to improve

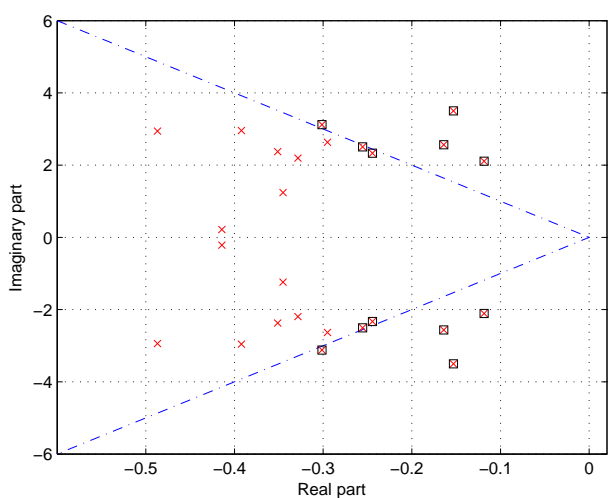

Fig. 2. Case 1: eigenvalues of the linear system (7) (crosses) and of the reduced order model (4) (squares). The dash-dotted lines represent the $10 \%$ damping ratio.

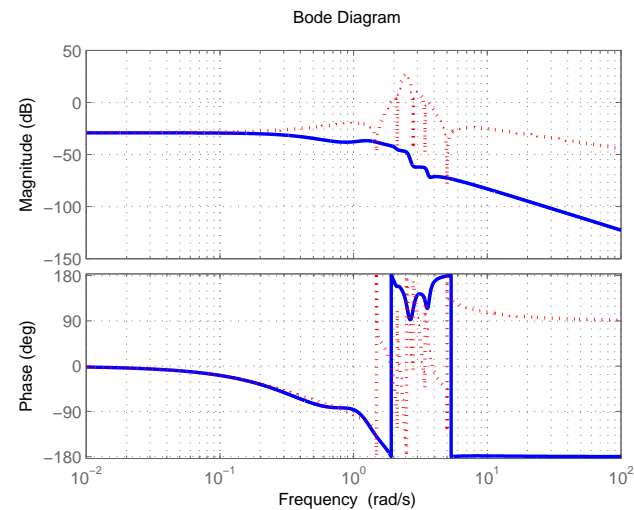

Fig. 3. Bode plot (2nd diagonal term of the transfer function) of the linear system (7) (solid lines) and of the reduced order model (4) (dotted lines) for the set of eigenvalues shown in Fig.2.

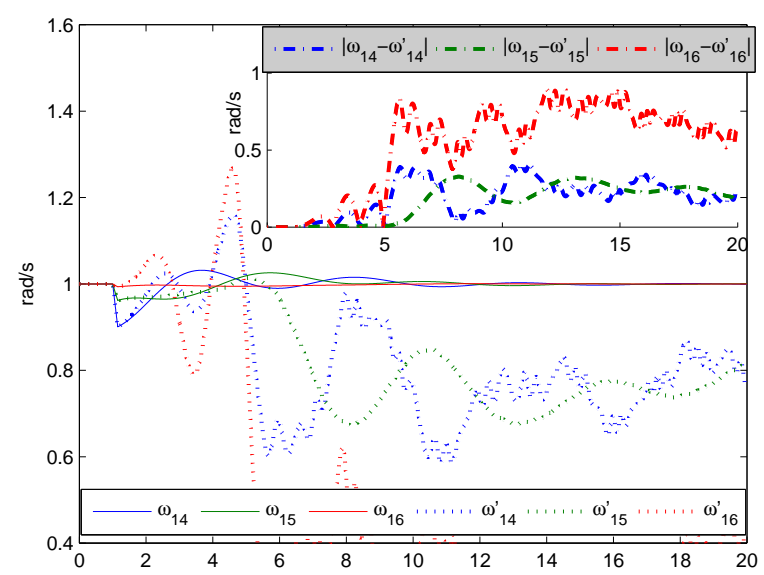

Fig. 4. Large: angular velocities of the study area when this is connected to the nonlinear system describing the external area (solid lines) and when it is connected to the reduced order model (dotted lines) with the eigenvalues shown in Fig.2. Small: absolute errors between the time histories. 


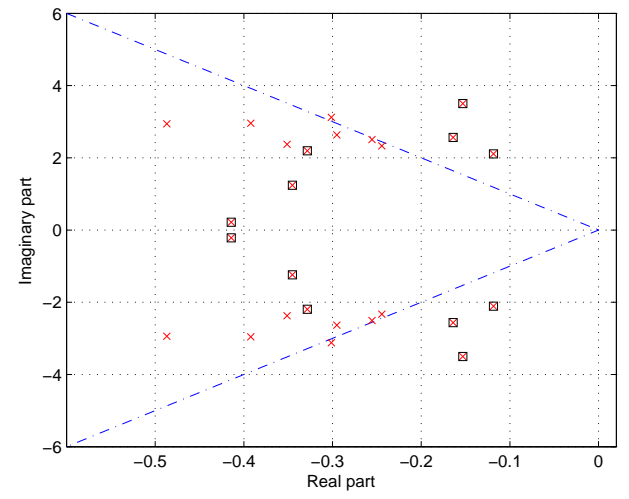

Fig. 5. Case 2: eigenvalues of the linear system (7) (crosses) and of the reduced order model (4) (squares). The dash-dotted line represent the $10 \%$ damping ratio.

the approximation it is necessary to increase the number of interpolation points or, equivalently, the dimension of the reduced order model. Note that for each case that we consider we show only one randomly chosen Bode plot (for the sake of this paper, the 2 nd diagonal term of the transfer function) out of the total thirty-nine. In all cases, all thirty-nine Bode plots show similar behavior and they are omitted for this reason.

Case 1: we start with computing a reduced order model of dimension $\nu=12$. The interpolation points have been chosen to be at 0 (zero and first moment), at $0.234 \mathrm{~Hz}, 0.3374 \mathrm{~Hz}$, $0.444 \mathrm{~Hz}, 0.5443 \mathrm{~Hz}, 0.7958 \mathrm{~Hz}$ (all zero moments). Note that the solution of equation (2) can be computed with the function Sylvester of MATLAB. However, the result is very imprecise for MIMO systems. We suggest to implement a custom function based on the Kronecker product, see e.g. [1]. We determine the twelve least damped eigenvalues of system (7) and we assign them to the reduced order model (4). In Fig. 2 the eigenvalues of system (7) are represented with crosses, whereas the eigenvalues of the reduced order model are depicted with squares. In the figure the modes in the area between the two dash-dotted lines are well damped (more than $10 \%$ damping ratio), whereas the others are considered poorly damped. Fig. 3 shows the Bode plot of system (7) (solid lines) and of the reduced order model (dotted lines). We can see that the two graphs starts to diverge already around 0.0318 $\mathrm{Hz}$ and that the behavior from medium to high frequencies is very different. A dynamic simulation of the power system is performed. A self-clearing fault at bus 14 of the study area occuring at $t=1 \mathrm{~s}$ and cleared at $1.15 \mathrm{~s}$ is simulated. Fig. 4 shows the angular velocities (large) and respective absolute errors (small) of the study area when this is connected to the nonlinear system describing the external area (solid lines) and when it is connected to the reduced order model (dotted lines). The approximation given by the reduced order model is totally unsatisfactory. Although Fig. 3 shows a good approximation of the steady state (low frequency), the interconnection of the reduced order model and the study area is unstable. In fact, the fault generates high frequency oscillations which make the trajectories of the system to exit the region of attraction of the equilibrium point.

Case 2: the common approach used to improve the quality of the approximation is to increase the order of the reduced

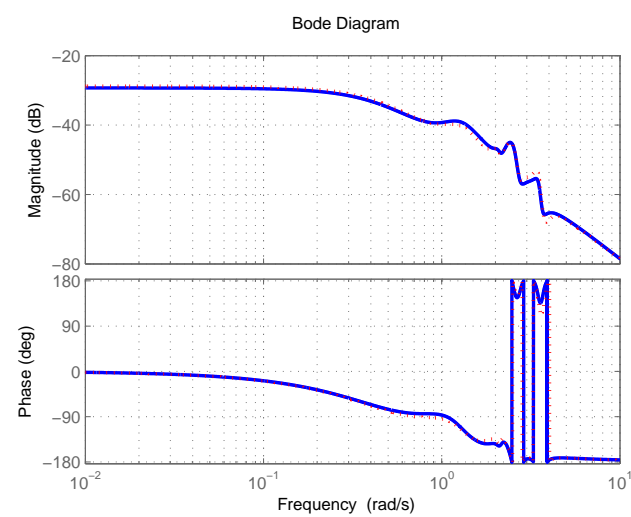

Fig. 6. Bode plot (2nd diagonal term of the transfer function) of the linear system (7) (solid lines) and of the reduced order model (4) (dotted lines) for the set of eigenvalues shown in Fig.5.

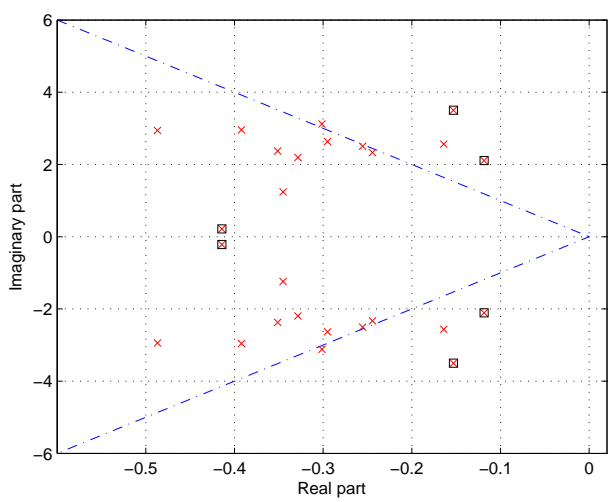

Fig. 7. Case 3: eigenvalues of the linear system (7) (crosses) and of the reduced order model (4) (squares). The dash-dotted lines represent the $10 \%$ damping ratio.

model in the hope that the new reduced order model be able to capture better the dynamics of the system to be reduced. We show that this is not necessary. We keep the same order $\nu=12$ maintaining the same matrices $S$ and $L$. This time we assign as eigenvalues the six most poorly damped modes and the six slowest modes. Fig. 5 shows the eigenvalue selection for this case. Fig. 6 shows the Bode plot of system (7) (solid lines) and of the reduced order model (dotted lines). We note that the two graphs are close. The dynamic simulation graph for this case is omitted since it is similar to the next, more interesting, case. Thus we see that the eigenvalues retained in the reduced order model play a role that can be more important of the order of the reduction.

Case 3: to strengthen this last observation we now decrease the order of the reduced model to $\nu=6$. The interpolation points have been chosen as a subset of the previous case, namely 0 (zero and first moment), $0.3374 \mathrm{~Hz}$ and 0.5443 $\mathrm{Hz}$ (all zero moments). This time we maintain the four most poorly damped modes and the two slowest modes. Fig. 7 shows the eigenvalue selection for this case. Fig. 8 shows the Bode plot of system (7) (solid lines) and of the reduced order model (dotted lines). We see that in this case the reduced order model is a better approximation with respect to case 1 ( $\nu=12$ with a bad selection of eigenvalues) but obviously 


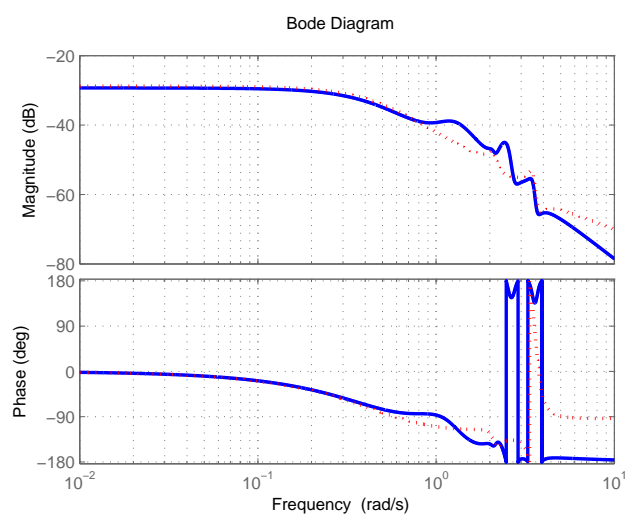

Fig. 8. Bode plot (2nd diagonal term of the transfer function) of the linear system (7) (solid lines) and of the reduced order model (4) (dotted lines) for the set of eigenvalues shown in Fig.7.

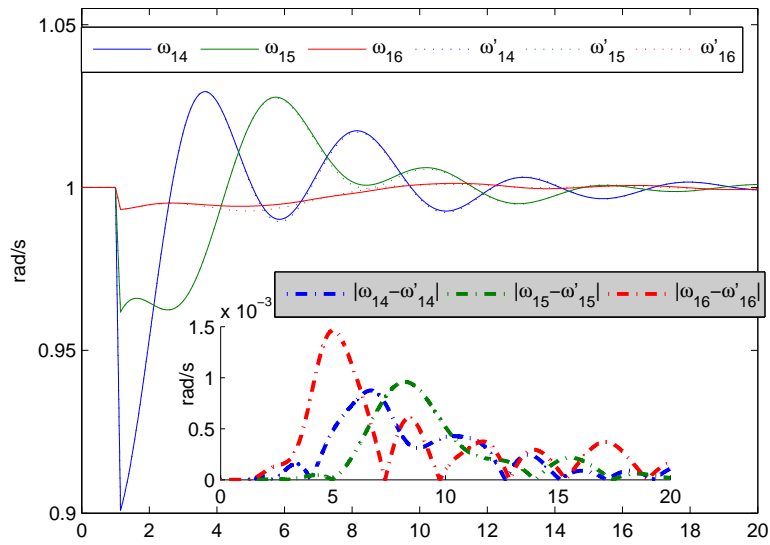

Fig. 9. Large: angular velocities of the study area when this is connected to the nonlinear system describing the external area (solid lines) and when it is connected to the reduced order model (dotted lines) with the eigenvalues shown in Fig.7. Small: absolute errors between the time histories.

a worse approximation with respect to case $2(\nu=12$ with a good selection of eigenvalues). However, for the dynamic behavior of interest this approximation is sufficiently good. In fact, Fig. 9 shows the angular velocities (large) and respective absolute errors (small) of the study area when this is connected to the nonlinear system describing the external area (solid lines) and when it is connected to the reduced order model (dotted lines). We see that the two time histories are almost indistinguishable.

\section{CONCLUSiON}

We have presented a moment matching model reduction technique which can be used to easily assign arbitrary eigenvalues to the reduced order model. We have exploited the possibility to preserve slow and poorly damped modes. We have shown that it is not necessary to increase the order of the reduced order model to improve the quality of the approximation. In particular we have shown that increasing the order of the reduced model gives no guarantee of improving the quality of the approximation if the wrong set of eigenvalues is preserved. Note that other implications of the method presented in [10] may be of interest in power systems. In particular the nonlinear model reduction and the ideas presented in [14].

\section{ACKNOWLEDGMENT}

The author would like to thank Prof. A. Astolfi for his constant help and advice.

\section{REFERENCES}

[1] A. Antoulas, Approximation of Large-Scale Dynamical Systems. Philadelphia, PA: SIAM Advances in Design and Control, 2005.

[2] J. H. Chow, Power System Coherency and Model Reduction, ser. Power Electronics and Power Systems. Springer, 2013.

[3] A. J. Germond and R. Podmore, "Dynamic aggregation of generating unit models," IEEE Transactions on Power Apparatus and Systems, vol. PAS-97, no. 4, pp. 1060-1069, July 1978.

[4] R. Podmore, "Identification of coherent generators for dynamic equivalents," IEEE Transactions on Power Apparatus and Systems, vol. PAS97, no. 4, pp. 1344-1354, July 1978.

[5] B. Avramovic, P. V. Kokotovic, J. R. Winkelman, and J. H. Chow, "Area decomposition for electromechanical models of power systems," Automatica, vol. 16, no. 6, pp. 637-648, 1980.

[6] P. V. Kokotovic, B. Avramovic, J. H. Chow, and J. R. Winkelman, "Coherency based decomposition and aggregation," Automatica, vol. 18, no. 1, pp. 47-56, 1982.

[7] D. Chaniotis and M. A. Pai, "Model reduction in power systems using Krylov subspace methods," IEEE Transactions on Power Systems, vol. 20, no. 2, pp. 888-894, May 2005.

[8] C. Sturk, L. Vanfretti, Y. Chompoobutrgool, and H. Sandberg, "Coherency-independent structured model reduction of power systems," IEEE Transactions on Power Systems, vol. 29, no. 5, pp. 2418-2426, Sept 2014.

[9] A. K. Singh and B. C. Pal, "Report on the 68-bus, 16-machine, 5-area system," IEEE PES Task Force on Benchmark Systems for Stability Controls. Ver. 3.3, Dec [Online] http://www.sel.eesc.usp.br/ieee/, 2013.

[10] A. Astolfi, "Model reduction by moment matching for linear and nonlinear systems," IEEE Transactions on Automatic Control, vol. 55, no. 10, pp. 2321-2336, 2010.

[11] G. Scarciotti and A. Astolfi, "Model reduction by moment matching for linear time-delay systems," 19th IFAC World Congress, Cape Town, South Africa, August 24-29, 2014.

[12] _ - "Model reduction by moment matching for nonlinear time-delay systems," in Proceedings of the 53rd IEEE Conference on Decision and Control, 2014.

[13] _ "Characterization of the moments of a linear system driven by explicit signal generators," in Proceedings of the 2015 American Control Conference, Chicago, IL, July, 2015.

[14] _ "Model reduction for linear systems and linear time-delay systems from input/output data," in 2015 European Control Conference, Linz, July, 2015.

[15] K. Gallivan, A. Vandendorpe, and P. Van Dooren, "Sylvester equations and projection-based model reduction," Journal of Computational and Applied Mathematics, vol. 162, no. 1, pp. 213-229, 2004.

[16] K. A. Gallivan, A. Vandendorpe, and P. Van Dooren, "Model reduction and the solution of Sylvester equations," in MTNS, Kyoto, 2006.

[17] T. C. Ionescu and A. Astolfi, "Families of moment matching based, structure preserving approximations for linear port Hamiltonian systems," ArXiv e-prints, Apr 2013.

[18] T. C. Ionescu, A. Astolfi, and P. Colaneri, "Families of moment matching based, low order approximations for linear systems," Systems \& Control Letters, vol. 64, pp. 47-56, 2014.

[19] K. A. Gallivan, A. Vandendorpe, and P. Van Dooren, "Model reduction of MIMO systems via tangential interpolation," SIAM Journal on Matrix Analysis and Applications, vol. 26, no. 2, pp. 328-349, 2004.

[20] P. Sauer and A. Pai, Power System Dynamics and Stability. Prentice Hall, 1998.

[21] P. M. Anderson and A. A. A. Fouad, Power system control and stability, 2nd ed., ser. Power Engineering Series. IEEE Press, 2003. 